\title{
Satisfacción materna con manejo del dolor en posparto quirúrgico en un hospital del norte de México
}

\section{Maternal satisfaction with postpartum surgical pain management in a hospital in northern Mexico}

\section{Satisfação materna com a gestão da dor cirúrgica pós-parto num hospital do norte do México}

\author{
Julia Teresa López-España ${ }^{\text {* }}$ \\ (D)https://orcid.org/0000-0003-3378-4940 \\ María Elena Calderón-Dimas ${ }^{2}$ \\ (iDhttps://orcid.org/0000-0002-3721-4796 \\ María Guadalupe Moreno-Monsiváis ${ }^{3}$ \\ (D)https://orcid.org/0000-0002-7152-00244 \\ Ma. Guadalupe Interial-Guzmán ${ }^{4}$ \\ (iDhttps://orcid.org/0000-0003-1231-4077
}

1. Jefe de Servicios Académicos para el Aprendizaje del Profesor y Estudiante, Facultad de enfermería, Universidad Autónoma de Nuevo León, Monterrey, Nuevo León México

2. Jefe de Departamento, Instituto Mexicano del Seguro Social Monterrey, Nuevo León México

3. Directora de Facultad de Enfermería, Universidad Autónoma de Nuevo León, Monterrey, Nuevo León México

4. Secretaría Académica del Pregrado, Facultad de Enfermería, Universidad Autónoma de Nuevo León, Monterrey, Nuevo León México

*Autor para correspondencia: july0521@hotmail.com

Recibido: 08/06/2020

Aceptado: 17/09/2021 


\title{
Resumen
}

Introducción: La calidad de la atención es una prioridad en el sistema de salud en especial la satisfacción del paciente. El manejo del dolor postoperatorio es un indicador que puede afectarlo Objetivo: Establecer la relación entre satisfacción materna con el manejo del dolor postoperatorio, con intensidad del dolor, orientación preoperatoria, tiempo de respuesta del personal de salud y factores obstétricos en un hospital del norte de México. Metodología: Diseño descriptivo, correlacional. Participaron 259 con posparto quirúrgico de un hospital público de Monterrey, Nuevo León, México. Se utilizó el Cuestionario de Intensidad del dolor en el paciente: Se incluyó en el estudio a mujeres con 24 horas de posparto quirúrgico; se excluyó a mujeres con salpingoclasia o histerectomía. Se utilizó estadística descriptiva e inferencial.

Resultados: Todas las participantes presentaron dolor, $61.4 \%$ refirió dolor severo en las primeras 24 horas, $69.5 \%$ señaló un tiempo de respuesta para manejo del dolor menor a 10 minutos. La media de satisfacción fue $8.9(D E=1.3)$. Se encontró relación entre la satisfacción materna con el manejo del dolor postoperatorio y el tiempo de respuesta del personal ( $p=.001)$; así como con el número de cesáreas $(p=.04)$. Las mujeres que recibieron orientación preoperatoria reportaron mayor satisfacción con el manejo del dolor (9 vs 8.27, $p=.001$ ) y menor dolor (7.87 vs $6.90, p=.01$ ). Conclusiones: El correcto manejo del dolor debe priorizarse como criterio de calidad. La orientación preoperatoria es una práctica que debe reforzarse en la atención a pacientes quirúrgicos.

Palabras clave: Período Posparto; Satisfacción del Paciente; Dolor Postoperatorio (DeCS).

\begin{abstract}
Introduction: The quality of care is a priority in the health system, especially patient satisfaction. Postoperative pain management is an indicator that can affect it. Objective: Establish the relationship between maternal satisfaction with postoperative pain management, pain intensity, preoperative orientation, health personnel response time and obstetric factors in a hospital in northern Mexico. Methodology: Descriptive, correlational design. A total of 259 postpartum surgical patients from a public hospital in Monterrey, Nuevo Leon, Mexico participated. The Patient Pain Intensity Questionnaire was used; women with 24 hours of postpartum surgery were included in the study; women with tubal ligation or hysterectomy were excluded. Descriptive and inferential statistics were used. Results: All participants presented pain; $61.4 \%$ reported severe pain in the first 24 hours; $69.5 \%$ reported a response time for pain management of less than 10 minutes. Mean satisfaction was $8.9(S D=1.3)$. A relationship was found between maternal satisfaction with postoperative pain management and personnel response time $(p=.001)$; as well as with the number of cesarean sections $(p=.04)$. Women who received preoperative counseling reported greater satisfaction with pain management ( 9 vs. $8.27, p=.001)$ and less pain (7.87 vs. 6.90, $p=.01$ ). Conclusions: Correct pain management should be prioritized as a quality criterion. Preoperative orientation is a practice that should be reinforced in the care of surgical patients.
\end{abstract}

Key words: Postpartum Period; Patient Satisfaction; Postoperative Pain (DeCS).

\begin{abstract}
Abstrato
Introdução: A qualidade do atendimento é prioridade no sistema de saúde, principalmente a satisfação do paciente. O manejo da dor pós-operatória é um indicador que pode afetá-la. Objetivo: Estabelecer a relação entre a satisfação materna com o manejo da dor pós-operatória, intensidade da dor, orientação pré-operatória, tempo de resposta do pessoal de saúde e fatores obstétricos em um hospital no norte do
\end{abstract}


México. Metodologia: Desenho descritivo e correlacional. Um total de 259 pacientes cirúrgicas pós-parto de um hospital público em Monterrey, Nuevo Leon, México participaram. O Questionário de Intensidade da Dor do Paciente foi usado; mulheres com 24 horas de cirurgia pós-parto foram incluídas no estudo; mulheres com laqueadura ou histerectomia foram excluídas. Estatísticas descritivas e inferenciais foram utilizadas. Resultados: Todos os participantes apresentaram dor; $61,4 \%$ relataram dor intensa nas primeiras 24 horas; 69,5\% relataram um tempo de resposta para o controle da dor de menos de 10 minutos. A satisfação média foi de $8,9(\mathrm{DP}=1,3)$. Foi encontrada uma relação entre a satisfação materna com o manejo da dor pós-operatória e o tempo de resposta do pessoal $(p=0,001)$; bem como com o número de cesáreas $(p=0,04)$. Mulheres que receberam aconselhamento pré-operatório relataram maior satisfação com o controle da dor (9 vs. 8,27, $p=0,001)$ e menos dor $(7,87$ vs. $6,90, p=0,01)$. Conclusões: O gerenciamento correto da dor deve ser priorizado como um critério de qualidade. A orientação préoperatória é uma prática que deve ser reforçada no cuidado ao paciente cirúrgico.

Palavras-chave: Período Pós-Parto; Satisfação do Paciente; Dor Pós-Operatória (DeCS).

\section{Introducción}

La calidad es un requisito esencial en la atención que otorga el personal de salud, demanda una entrega de cuidado accesible, individualizada y cálida, permanente y eficiente, el objetivo es satisfacer las necesidades del usuario y de los prestadores que brindan la atención. Un tema que tiene mayor interés en la gestión de la salud es la satisfacción del paciente ${ }^{(1)}$. Esta puede verse afectada por diversos factores, uno de los reportados en la literatura es el dolor, específicamente su manejo ${ }^{(2)}$. Para este estudio es prioritario el dolor de tipo postoperatorio por ser uno de los más comunes ante la alta demanda de procedimientos quirúrgicos $(3-5)$.

Una de las mayores demandas de atención en México es la salud reproductiva, la cual se enfatiza en la atención durante la gestación, parto y post parto. En México el $49.7 \%$ del total de mujeres corresponde a edad fértil, con una tasa de natalidad del 18.5 por cada mil habitantes ${ }^{(6)}$. Es esperado que estos embarazos culminen con un parto; sin embargo, un $46.3 \%$ termina en parto quirúrgico, por lo que se incrementa al interior de los servicios de salud la demanda de cuidados postoperatorios en este grupo poblacional (7).

Algunos autores destacan que el dolor postoperatorio, suele tratarse de manera incorrecta e insuficiente. Lo anterior es confirmado en estudios, donde se reporta que alrededor del $95 \%$ de las mujeres 
con posparto quirúrgico expresan dolor postoperatorio moderado, intenso o extremo ${ }^{(8-10)}$. Las consecuencias de un mal manejo del dolor, en el puerperio quirúrgico pueden desencadenar un aumento en complicaciones respiratorias y tromboembólicas ocasionadas por la disminución de movimiento de las mujeres, por efecto del dolor ${ }^{(11,12)}$.

Esto impacta negativamente debido a que las pacientes tienen dificultad al deambular tempranamente y durante su estadía en el hospital, lo que incrementa su insatisfacción e interfiere con su relación madre-hijo en los primeros días de la vida del recién nacido, debido a que el dolor le dificulta cargarlo y atenderlo, alimentarlo con lactancia materna, produciendo ansiedad y depresión posparto (13-16).

La satisfacción con el manejo del dolor postoperatorio ha sido estudiada por diferentes autores, quienes reportan que, a mayor orientación preoperatoria, mayor satisfacción con el manejo del dolor $(2,15)$. Así mismo, otro estudio muestra que, a mayor dolor reportado, menor satisfacción (2), sin embargo, en el caso del período posparto, las pacientes a pesar de que se presenta el dolor, el nivel de satisfacción es alta (15-19). En otro estudio realizado en España en mujeres intervenidas de cesárea donde se calificó el dolor postoperatorio, la intensidad reportó media de 3.66, imposibilitando al $58 \%$ de las mujeres a efectuar actividades como sentarse en la cama o toser. Sin embargo, $41 \%$ de las participantes que recibieron información preoperatoria, refirieron mayor satisfacción con el manejo del dolor postoperatorio (20).

Otros autores concluyen que el dolor que se experimenta en el posoperatorio de la cesárea, es la consecuencia de una experiencia única ocasionada por muchos factores, entre ellos la ansiedad, los aspectos étnicos y culturales, la cesárea previa, las mujeres jóvenes, el tipo de incisión de cesárea y el contexto, además del estado de salud del recién nacido (21-22).

En otro estudio, destacan que la atención inmediata o la brevedad del personal sanitario a la necesidad de dolor después de la cirugía, es importante para la satisfacción del paciente, pues un alto porcentaje de pacientes (83.9\%) que recibieron la analgesia solicitada en menos de 15 minutos, demostraron mayor satisfacción con el manejo del dolor postoperatorio ${ }^{(23)}$. 
De lo descrito anteriormente, puede apreciarse que la satisfacción se ha asociado con el dolor y su intensidad, así como con experiencia previa, orientación recibida, tiempo de respuesta o percepción de un manejo oportuno ante la presencia de dolor, y en el caso de mujeres en el posparto quirúrgico se ha asociado con el tipo de incisión quirúrgica. Sin embargo, los hallazgos no son conclusivos y en este grupo en particular los resultados parecen diferir, mientras que para unos, la intensidad del dolor disminuye, en otros la satisfacción del cuidado es alta.

Teniendo en cuenta estos antecedentes, esta investigación tuvo como objetivo establecer la relación entre satisfacción materna con el manejo del dolor postoperatorio, intensidad del dolor, orientación preoperatoria, tiempo de respuesta del personal de salud y factores obstétricos.

\section{Metodología}

Estudio descriptivo y correlacional. La población correspondió a pacientes durante el período de posparto quirúrgico, hospitalizadas en el departamento de obstetricia en una institución pública de seguridad social, ubicada en Monterrey, Nuevo León, México. Para la selección de pacientes, se utilizó un muestreo sistemático de uno en dos con un inicio aleatorio, se eligió al azar a la primera participante y posteriormente a una de cada dos pacientes. Se incluyó en el estudio a mujeres con un día completo de posparto quirúrgico, sin complicaciones en su evolución, ni con la salud del recién nacido; se excluyó a las que además de la cesárea, les realizaron otro procedimiento quirúrgico como salpingoclasia o histerectomía.

Para estimar el tamaño de la muestra se utilizó el paquete estadístico nQuery advisor 4.0 con una correlación de .20 , un nivel de significancia de .05 y un poder de $90 \%$. Se obtuvo una muestra de 259 participantes. La recolección de datos se realizó de Enero a Marzo de 2016.

Para medir las variables del estudio: satisfacción materna con el manejo del dolor postoperatorio, intensidad del dolor, orientación preoperatoria, tiempo de respuesta del personal de salud y factores obstétrico, se utilizó el Cuestionario de Intensidad del Dolor en el Paciente (CIDP) ${ }^{(24)}$, este instrumento mide la intensidad del dolor y el nivel de satisfacción con el manejo del dolor por parte del personal de enfermería 
y médico. El cuestionario ha obtenido una alfa de Cronbach, de .85 , lo que significa que es confiable. Para fines de este estudio se utilizaron solo nueve ítems, que miden la intensidad y presencia del dolor, mediante una escala numérica visual, con evaluaciones que van de una puntuación de 0 a 10. La intensidad se clasificó como ausencia de dolor (valores de cero), dolor leve (1-3), dolor moderado (4-6) y dolor severo (7-10).

Así mismo, contiene una pregunta que valora la interrupción cuando hay dolor en diversas actividades como el sueño y el humor, con puntajes de 0 a $10(0=$ ausencia de interrupción y $10=$ Alta interrupción). Para evaluar la orientación otorgada a la mujer para el tratamiento del dolor, un ítem evalúa con respuesta dicotómica (Sí/No) si le brindaron orientación. Finalmente, para evaluar 1a la satisfacción con el tratamiento del dolor en el posparto quirúrgico, como resultado de la atención brindada por el personal de enfermería y médico, se aplicaron dos preguntas con opciones de respuesta de 0 a 10 ( 0 puntos= ausencia total de satisfacción y 10 puntos= la más alta satisfacción). El tiempo de respuesta para recibir el analgésico solicitado se midió con opciones de menor a 10 minutos hasta 60 minutos.

Referente a los aspectos éticos, este estudio consideró el Reglamento de la Ley General de Salud en Materia de Investigación ${ }^{(25)}$, y fue avalado por los Comités de Ética y de Investigación de la Facultad de Enfermería de la Universidad Autónoma de Nuevo León y de la institución de salud participante (FAEN-M1506).

A todos los participantes se les solicitó su autorización y la firma del consentimiento informado. Durante la recolección de los datos se cuidó no interferir con la atención. Si la paciente refirió dolor durante la aplicación del instrumento, se canalizó al personal de enfermería para su manejo y después se regresó con la paciente para continuar con el cuestionario.

La captura y el análisis de los datos fue a través del paquete estadístico Statistical Package for the Social Sciences (SPSS) versión 20. El análisis inferencial fue a través de pruebas no paramétricas debido a que los datos mostraron distribución no normal mediante la prueba de Kolmogorov Smirnov. Para 
comprobar la relación de las variables propuestas se aplicó la correlación de Spearman y la U de MannWhitney para identificar diferencia entre los grupos.

\section{Resultados}

Las participantes reportaron una media de 26 años de edad $(D E=5.09)$ y 10 años $(D E=2.57)$ de escolaridad, lo que significa tener una preparación de preparatoria incompleta. La media de gestaciones fue de dos y el 50.2\% tenía antecedente de cesárea previa.

El dolor máximo que refirieron las mujeres en las primeras 24 horas, se ubicó en una media de 7 ( $D E=2.56)$ y el reporte en el momento de la entrevista, obtuvo una media de $4.45(D E=1.81)$. Al clasificar la intensidad del dolor predominó el dolor moderado, mientras que el dolor máximo presentado en las primeras 24 horas fue clasificado como severo, en $61.4 \%$ de las participantes, el dolor promedio fue calificado como moderado por $68 \%$ (Tabla1).

Tabla 1. Distribución de mujeres según la clasificación del nivel de dolor durante el puerperio quirúrgico

\begin{tabular}{ccccccc}
\hline $\begin{array}{c}\text { Intensidad del } \\
\text { Dolor }\end{array}$ & $\begin{array}{c}\text { Dolor al momento de } \\
\text { la entrevista }\end{array}$ & \multicolumn{2}{c}{$\begin{array}{c}\text { Dolor máximo en las } \\
\text { primeras } 24 \text { horas }\end{array}$} & $\begin{array}{c}\text { Nivel promedio de dolor en las } \\
\text { primeras 24 horas }\end{array}$ \\
\cline { 2 - 7 } & $f$ & $\%$ & $f$ & $\%$ & $f$ & $\%$ \\
Leve & 86 & 33.2 & 32 & 12.4 & 42 & 16.0 \\
Moderado & 133 & 51.4 & 68 & 26.2 & 175 & 68.0 \\
Severo & 40 & 15.4 & 159 & 61.4 & 42 & 16.0 \\
\hline Fuente: Cuestionario de Intensidad del Dolor en el Paciente & & &
\end{tabular}

La media obtenida de la satisfacción global del manejo del dolor por parte del personal de salud, fue de 8.9 $(D E=1.30)$. El personal de enfermería obtuvo una media ligeramente mayor respecto al personal médico (9.02 vs 8.87) (Tabla 2). 
Tabla 2. Opinión de las mujeres de la satisfacción con el manejo del dolor por parte del personal de salud.

\begin{tabular}{lccccc}
\hline \multicolumn{1}{c}{ Satisfacción de la mujer } & Media & Mediana & $\begin{array}{c}\text { Desviación } \\
\text { Estándar }\end{array}$ & $\begin{array}{c}\text { Valor } \\
\text { Mínimo }\end{array}$ & $\begin{array}{c}\text { Valor } \\
\text { Máximo }\end{array}$ \\
\hline Con el manejo del dolor por parte del médico & 8.87 & 9.00 & 1.56 & 0 & 10 \\
$\begin{array}{l}\text { Con el manejo del dolor por parte de la } \\
\text { enfermera }\end{array}$ & 9.02 & 9.00 & 1.27 & 0 & 10 \\
\begin{tabular}{l} 
Satisfacción global \\
\hline
\end{tabular} & 8.90 & 9.00 & 1.30 & 2 & 10 \\
\hline
\end{tabular}

Fuente: Cuestionario de Intensidad del Dolor en el Paciente

$n=259$

El $81.1 \%$ de las mujeres, refirieron solicitar analgésico cuando presentó dolor, y el promedio del tiempo de respuesta para recibirlo fue menor de 10 minutos en el 69.5\% (Tabla 3).

Tabla 3. Distribución, según las mujeres del estudio, sobre el tiempo de respuesta para recibir el analgésico solicitado, por el personal de salud.

\begin{tabular}{lcc}
\hline \multicolumn{1}{c}{ Tiempo de respuesta al dolor } & $f$ & $\%$ \\
\hline Nunca solicité medicamentos para el dolor & 49 & 18.9 \\
Menos de 10 minutos & 180 & 69.5 \\
11 a 20 minutos & 24 & 9.3 \\
21 a 30 minutos & 4 & 1.5 \\
31 a 60 minutos & 8 & 0.2 \\
\hline Fuente: Cuestionario de Intensidad del Dolor en el Paciente & & $n=259$
\end{tabular}

Nota: $f=$ frecuencia, $\%=$ Porcentaje

No se encontró relación estadísticamente significativa entre la satisfacción materna con el manejo del dolor por tiempo de respuesta de médicos y enfermería con la intensidad del dolor que refirieron en la entrevista, el máximo dolor en las 24 horas y dolor promedio; sin embargo, se obtuvo una relación negativa estadísticamente significativa entre la satisfacción materna con el manejo del dolor por el personal de enfermería, y el tiempo de respuesta $\left(r_{s}=-.19, p=.01\right)$, hallazgo similar en relación con la satisfacción con el 
manejo del dolor por el médico con el tiempo de respuesta $\left(r_{s}=-.13, p=.03\right)$, lo que indica, que a menor tiempo de respuesta por parte del personal de salud, mayor satisfacción de la mujer. Finalmente, cabe destacar que la satisfacción materna con el manejo del dolor se relacionó positivamente con el número de cesáreas $\left(r_{s}=.13, p=.03\right)$, lo que significa que las mujeres que tenían mayor número de cesáreas reportaron una satisfacción mayor, estadísticamente significativa, con el manejo del dolor (Tabla 4).

Tabla 4. Cuadro de Correlación entre la satisfacción materna con el manejo del dolor y tiempo de respuesta, dolor máximo a las 24 horas y número de cesáreas

\begin{tabular}{lcc}
\hline Satisfacción materna con el manejo del dolor & $r$ & $p$ \\
\hline Tiempo de respuesta por parte de enfermería & -.19 & .01 \\
Tiempo de respuesta por parte del médico & -.13 & .03 \\
Dolor máximo a las 24 horas & .05 & .83 \\
Número de cesáreas & .12 & .04 \\
\hline Fuente: Cuestionario de Intensidad del Dolor en el Paciente & & $n=259$
\end{tabular}

La orientación preoperatoria la recibió el $82.62 \%$ de las mujeres, y uno de los hallazgos más destacado fue identificar diferencia estadísticamente significativa en la satisfacción materna con el manejo del dolor respecto a la orientación preoperatoria recibida. Las mujeres que recibieron orientación preoperatoria estuvieron más satisfechas que las que no la recibieron $(p=.001)$ (Tabla 5$)$.

Tabla 5. Diferencia entre la satisfacción percibida de las mujeres con puerperio quirúrgico sobre el manejo del dolor según la orientación preoperatoria.

\begin{tabular}{lccccc}
\hline \multicolumn{1}{c}{$\begin{array}{c}\text { Satisfacción con el manejo } \\
\text { del dolor }\end{array}$} & Media & Mediana & $\begin{array}{c}\text { Desviación } \\
\text { Estándar }\end{array}$ & U & p \\
\hline Recibieron orientación & 9.00 & 9 & 1.46 & 3605 & .001 \\
No recibieron orientación & 8.27 & 9 & 1.88 & & \\
\hline
\end{tabular}

Fuente: Cuestionario de Intensidad del Dolor en el Paciente

$n=259$ 
En las mujeres que reportaron un dolor de intensidad severo como dolor máximo en las primeras 24 horas, se analizó respecto a si recibieron o no orientación preoperatoria, Las mujeres que recibieron orientación señalan menor intensidad del dolor respecto a las que no recibieron (Media $=6.90$ vs 7.87 ). Las mujeres que no recibieron orientación refirieron que el dolor, interfirió más en sus actividades (Media= 3.64 vs 4.38)

\section{Discusión}

Un indicador o criterio de calidad en todos los sistemas sanitarios es el manejo oportuno y adecuado del dolor; sin embargo, los hallazgos del presente estudio muestran que todas las pacientes refirieron dolor en el puerperio quirúrgico y que la mayoría manifestó en las primeras 24 horas en el postoperatorio, un nivel entre moderado a intenso. Este hallazgo, coincide con lo referido por diversos autores $(2,8,9-12,19,20,22)$, quienes señalan que no son suficientes las acciones que se realizan para tratar el dolor. Este resultado es relevante debido a las limitaciones que genera y su efecto en la morbilidad de quien lo padece.

A pesar de lo anterior, la opinión del manejo del dolor obtuvo una satisfacción alta, este dato coincide con lo referido en otros estudios realizados en mujeres con características similares a las del

presente estudio ${ }^{(2,9,15-18)}$. Lo anterior evidencia qué en este grupo particular de participantes, la satisfacción se explica a través de otros aspectos, como es el caso de la orientación preoperatoria. Las mujeres que reportaron mayor satisfacción con el manejo del dolor por parte del personal médico y de enfermería fueron quienes recibieron orientación preoperatoria, este resultado fue similar con lo señalado por García ${ }^{(2,19,20)}$. La orientación que reciben las personas de lo que va a ocurrir después de una cirugía puede producir un efecto en las expectativas de los pacientes; para las participantes en el estudio, el conocer que posterior a un evento quirúrgico se presenta dolor y que se requiere recibir un tratamiento, les permite solicitar el medicamento prescrito para aliviar el dolor oportunamente con efecto en la disminución de la intensidad del dolor.

Es importante, buscar otras causas que expliquen ese hallazgo de aumento de dolor y mayor satisfacción, debido que, en otro estudio (2), se incluyeron pacientes con dolor postoperatorio, pero no en puerperio 
quirúrgico y ellos manifestaron menor dolor y mayor satisfacción. Lo anterior refleja que los hallazgos no son conclusivos y se requiere indagar el comportamiento de las variables en otros procedimientos quirúrgicos.

Otros aspectos relevantes que se relacionaron con la satisfacción con el manejo del dolor fueron el tiempo de respuesta del personal para el alivio del dolor, así como el número de cesáreas. Se identificó que las mujeres con menor tiempo de espera para recibir analgésico indicaron una mayor satisfacción, coincidiendo con otros autores ${ }^{(2,19,20)}$. Es claro que si se atiende de forma oportuna a las personas cuando tienen dolor, la satisfacción será mayor.

Se encontró que las mujeres que reportaron un mayor número de cesáreas previas reportaron una mayor satisfacción con el manejo del dolor, este hallazgo puede atribuirse a la experiencia previa, las mujeres saben que presentarán dolor, ya tienen una expectativa para su manejo por lo que las intervenciones pueden ser evaluadas comparando la experiencia previa. Estos hallazgos deben ser considerados en las intervenciones dirigidas al control del dolor.

La estandarización del manejo del dolor postoperatorio que incluya la orientación preoperatoria y el tiempo de respuesta para atender la presencia de dolor es una estrategia que sería relevante y útil, para mejorar la satisfacción de las mujeres. Es importante que estos aspectos sean considerados independientemente del número de embarazo de la mujer, sin embargo, es relevante enfatizar la orientación a las mujeres primigestas, debido a que no cuentan con la experiencia previa. El atender estos aspectos contribuirá por un lado a disminuir el nivel de dolor en las mujeres al participar activamente en su manejo, por otro lado, contribuirá a incrementar la satisfacción con el manejo del dolor, ambos aspectos considerados indicadores o criterios de calidad al interior de las instituciones de salud.

\section{Conclusiones}

Todas las pacientes refirieron dolor en el posparto quirúrgico, una alta proporción reportó intensidad de moderada a severa en las primeras 24 horas. A pesar de ello, se evidenció un alto índice de satisfacción 
materna con el manejo del dolor postoperatorio. Las pacientes que recibieron orientación preoperatoria, que esperaron menor tiempo de respuesta del personal para atender el dolor y con mayor número de cesáreas estaban satisfechas con el manejo del dolor postoperatorio.

Al ser el manejo del dolor un indicador de calidad y ante los hallazgos del estudio, cobra relevancia que los gestores del cuidado de enfermería consideren estrategias que contribuyan favorablemente a reducir la presencia e intensidad del dolor en mujeres en el posparto quirúrgico.

\section{Limitaciones del estudio}

Una limitación del estudio se atribuye al aspecto metodológico en cuanto al diseño transversal lo cual limita la causalidad.

\section{Conflicto de intereses}

Los autores declaran no tener ningún tipo de conflicto e intereses en relación al artículo.

\section{Financiamiento}

Los autores declaran no haber recibido financiamiento para realizar este trabajo.

\section{Referencias bibliográficas}

1. Alasad J, Tabar NA, Aburuz E. Patient satisfaction with Nursing care. Measuring outcomes in an international setting. JONA [Internet]. 2015. [consultado 5 may 2016];45:563-568 Disponible en: DOI: 10.1097 / NNA.0000000000000264

2. Moreno $M$, Muñoz $M$, Interial $M$. Satisfacción con el manejo del dolor posoperatorio en pacientes hospitalizados. Aquichan [Internet]. 2014 [consultado 15 feb 2016];14:460-472. Disponible en: DOI: http://dx.doi.org/10.5294/aqui.2014.14.4.2

3. Gerbershagen H, Pogatzki-Zahn E, Aduckathil S, Peelen L, Kappen T, Wijck A, et al. Procedure-specific risk factor analysis for the development of severe postoperative pain. Anesthesiology. [Internet] 2014. [consultado 3 ene 2016];120:1237-1245. Disponible en: https://pubmed.ncbi.nlm.nih.gov/24356102/

4. Consejo de Salubridad General. Estándares para implementar el modelo en hospitales. [Internet] México; 2018. [consultado 21 ene 2019]. Disponible en: http://www.csg.gob.mx/descargas/pdf/certificacionestablecimientos/modelo de seguridad/hospitales/Estandares-Hospitales-Edicion2018.pdf

5. Joint Commission International. Pain management: A systems approach to improving quality and safety. [Internet] Illinois; 2015. [consultado 12 ene 2016]. Disponible en: 
https://www.jointcommissioninternational.org/-/media/jci/jci-documents/accreditation/hospital-andamc/jci-errata-standards-only 7th-ed-hospital.pdf

6. Instituto Nacional de Estadística y Geografía. Natalidad y fecundidad: Sección temas natalidad [Internet] 2016. [actualización jun 2016; consultado 2 jun 2016]. Disponible en: https://www.inegi.org.mx/temas/natalidad/

7. Instituto Nacional de Estadística y Geografía e Informatica. Encuesta Nacional de la Dinámica Demográfica . ENADID 2014. Principales resultados [Internet] 2014. [consultado 13 feb 2016]. Disponible en: https://www.inegi.org.mx/contenidos/programas/enadid/2014/doc/resultados enadid14.pdf

8. Ramos-Rangel GE, Ferrer-Zaccaro LE, Mojica-Manrique VL, La Rotta MG. Manejo analgésico durante el postoperatorio de cesárea: estrategias farmacológicas. Rev Colomb Anestesiol [Internet] 2017. [consultado 3 feb 2018 ];45:327-334. Disponible en: DOl: http://dx.doi.org/10.1016/j.rca.2017.08.001

9. Ruedas Y, García C. Intensidad del dolor agudo postoperatorio y la satisfacción del paciente con manejo del dolor. [Tesis de licenciatura]. Guatemala, Universidad San Carlos. 2018:1-77. Disponible en: http://biblioteca.usac.edu.gt/tesis/05/05 11091.pdf

10. Cooklin A, Amir L, Jarman J, Midwifery G, Cullinane M, Donath S. Maternal physical health symptoms in the first 8 weeks postpartum among primiparous Australian women. BIRTH issues in perinatal care. [Internet] 2015. [consultado 5 ene 2016]; 42(3):254-260. Disponible en: https://doi.org/10.1111/birt.12168

11. Chou R, Gordon DB, De Leon-Casasola OA, Rosenberg JM, Bickler S, Brennan T et al. Management of postoperative pain: a clinical practice guideline from the American Pain Society, the American Society of Regional Anesthesia and Pain Medicine, and the American Society of Anesthesiologists' Committee on Regional Anesthesia, Executive Committee, and Administrative Council. J Pain [Internet] 2016 [consultado 10 mar 2017];17(2): 131-157. Disponible en: DOI: 10.1016/j.jpain.2016.02.002

12. Suárez A. Incidencia del dolor crónico tras la intervención quirúrgica de cesárea. [Tesis Doctoral] España, Universidad Compultense de Madrid. 2018;1-197. Disponible en: https://eprints.ucm.es/50263/1/T40669.pdf

13. Domke R., Contreras V., Contreras F., Carboneli P. Manejo del dolor Agudo Postoperatorio en operación cesárea. Revist Chil Obst Ginecol [Internet] 2018. [consultado 5 ene 2019];83(6):635-642. Disponible en: http://dx.doi.org/10.4067/S0717-75262018000600635

14. Bijl R, Freeman L, Weijenborg P, Middeldorp J, Dahan A, Dorp E. A retrospective study on persistent pain after childbirth in the Netherlands. J. Pain Res [Inernet] 2016. [consultado 3 mar 2017];9:1-8. Disponible en: DOI: 10.2147 / JPR.S96850

15. Kisa S, Zeyneloğlu S. Opinions of women towards cesarean delivery and priority issues of care in the postpartum period. Appl Nurs Res [Internet] 2016. [consultado 10 mar 2017];30:70-75. Disponible en: https://doi.org/10.1016/j.apnr.2015.11.004

16. Lozano Meza E C. Nivel de Satisfacción de la puérpera post cesárea sobre el cuidado que Brinda la enfermera en el servicio de ginecobstetricia del Hospital Nacional Daniel Alcides Carrión. [Tesis de Posgrado] Lima. Universidad Nacional Mayor de San Marcos. Perú 2017. Disponible en: http://cybertesis.unmsm.edu.pe/bitstream/handle/20.500.12672/7307/Lozano me.pdf?sequence=1\&is Allowed =y

17. Maehara K, Mori E, Tsuchiya M, Iwata H, Sakajo A, Tamakoshi K. Factors affecting maternal confidence and satisfaction in older Japanese primiparae during postpartum hospital stay. International Journal of 
Nursing Practice [Internet] 2016. [consultado 4 ene 2017];1:14-21. Disponible en: http://onlinelibrary.wily.com/doi/epdf/10.1111/ijn.12435

18. Rodríguez S, De la Cruz J, Roldan L, Terukina R, Placencia M, Moncada R. Nivel de satisfacción de gestantes atendidas según el tipo de parto en la clínica Good Hope. Revista Facultad de Medicina Humana [Internet] 2017. [consultado 11 octubre 2018];17(1):19-25. Disponible en: https://doi.org/10.25176/RFMH.v17.n1.744

19. Unyen Pinedo LM. Satisfacción ante el manejo del dolor posoperatorio en el Hospital II SuárezAngamos [Tesis de Posgrado] Lima, Universidad Mayor de San Marcos, Perú, 2010 Disponible en: https://cybertesis.unmsm.edu.pe/bitstream/handle/20.500.12672/13416/Unyen_Pinedo_Lilian_Melissa 2010.pdf? sequence $=3$ \&isAllowed $=y$

20. Gracía I. Calidad percibida por las mujeres intervenidas de cesárea, ante el manejo del dolor postoperatorio en cirugía obstétrica. Revista Nure Investigación [Internet] 2004. [consultado 23 ene 2016];8:1-8. Disponible en: http://www.nureinvestigacion.es/OJS/index.php/nure/article/view/185/168

21. Niklasson B, Georgsson $S$, Segerdahl M y Blanck A. risk factors for persistent pain and its influence on maternal wellbeing after cesarean section. AOGS. [Internet] 2015; 94:622-628. Disponible en: http://obgyn.onlinelibrary.wiley.com/doi/pdf/10.1111/aogs.1263

22. Carvalho N, Costa B, Fortunato $\mathrm{CH}$, Covalante T, Silva B, Varanda L. Dolor postoperatorio en mujeres sometidas a cesáreas. Enfermería Global [Internet]. 2017. [consultado 18 mar 2018];48:354-366. Disponible: http://doi.org/10.6018/eglobal.16.4.267721

23. Gallego,J I., Rodríguez de la Torre, M.R., Vázquez, G. J, Gill,M. Estimación de la prevalencia e intensidad del dolor postoperatorio y su relación con la satisfacción de los pacientes. Rev Soc Esp Dolor [Internet] 2004. [consultado 2 ene 2016];11(4):197-202. Disponible en: http://scielo.isciii.es/scielo.php?script=sci arttext\&pid=S1134-80462004000400003

24. McCaffery M, Pasero C. Pain: Clinical Manual. St. Louis: Mosby. 1999.

25. Secretaría de Salud. Reglamento de la Ley General de Salud en Materia de Investigación para la Salud. México. 2014. [consultado 5 dic 2016]. Disponible en: https://www.dof.gob.mx/nota detalle.php?codigo $=5339162 \&$ fecha $=02 / 04 / 2014$

Cómo citar este artículo: López-España J, Calderón-Dimas ME, Moreno-Monsiváis MG, Interial-Guzmán MG. Satisfacción materna con manejo del dolor en posparto quirúrgico en un hospital del norte de México.

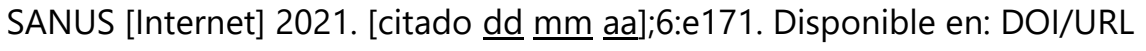

